\title{
Review of: "ACCURATE DECODING OF THE SPINAL CORD OUTPUT IN HUMANS WITH IMPLANTED HIGH- DENSITY ELECTRODE ARRAYS"
}

Ronald Knepper ${ }^{1}$

1 Boston University

Potential competing interests: The author(s) declared that no potential competing interests exist.

Review of "Accurate Decoding of the Spinal Cord Output in Humans with Implanted High-Density Electrode Arrays"

I want to congratulate the authors of this report for a very interesting paper submission and an impressive accomplishment in the field of neuroscience and bioengineering. Although much of the work presented in this paper is outside my main area of expertise, I feel that it is of considerable interest, a significant contribution to the field of interpreting neural signals, and is worthy of publication in bioRxiv. The ability to observe the action potential signals of 40 neurons within a space of $1-2 \mathrm{~cm}$ in a human muscle would seem to be of considerable value and would be useful for future work to improve our understanding of the behavior of motor neurons.

I have a few comments and suggestions that I will include below:

- Abbreviations such as EMG, MN, MU, BSS, MVC, CL, etc. should be included as part of the Keywords descriptions given on the first page.

- Page 5, line 100 - "synaptic input is a frequency range up to $75 \mathrm{~Hz}$ " should be modified to read "synaptic input at a frequency range up to $75 \mathrm{~Hz}$ ".

- Page 7, line 133 - I think the authors mean "Figure 1F", not "Figure 3F".

- The section entitled METHODS at the end of the paper includes so much low-necessity detail that it makes reading difficult. I don't think all this detail is necessary, but since it is tacked on at the end of the paper, I suppose it can be included as written.

- The section entitled DISCUSSION is also rather difficult to understand for someone who is not wellversed in this particular field of neuroscience and bioengineering. Much attention is given to expressing the success of the implanted high-density electrode arrays for measuring motor neurons, but little effort is made to explain, for example, how Figure 3 is obtained.

All in all, I believe this paper submission demonstrates an interesting and exciting approach for measuring and decoding spinal cord output in humans with an implanted high-density electrode array. I feel it is worthy of publication in bioRxiv. 
